\title{
Pancreas Contour Detection Based On Shearlet Domain In Low Quality Medical Images
}

\author{
Nguyen Thanh Binh ${ }^{1, *}$, Nguyen Mong Hien ${ }^{1,2}$, Pham Bao Quoc ${ }^{1}$, Vo Thi Hong Tuyet ${ }^{3}$ \\ ${ }^{1}$ Faculty of Computer Science and Engineering, Ho Chi Minh City University of Technology, VNU-HCM, Vietnam, \\ ntbinh@hcmut.edu.vn, nationpham@hotmail.com \\ ${ }^{2}$ Faculty of Engineering and Technology, Tra Vinh University, Vietnam, hientvu@tvu.edu.vn \\ ${ }^{3}$ Faculty of Information Technology, Ho Chi Minh City Open University, Vietnam, tuyet.vth@ou.edu.vn
}

\section{Abstract}

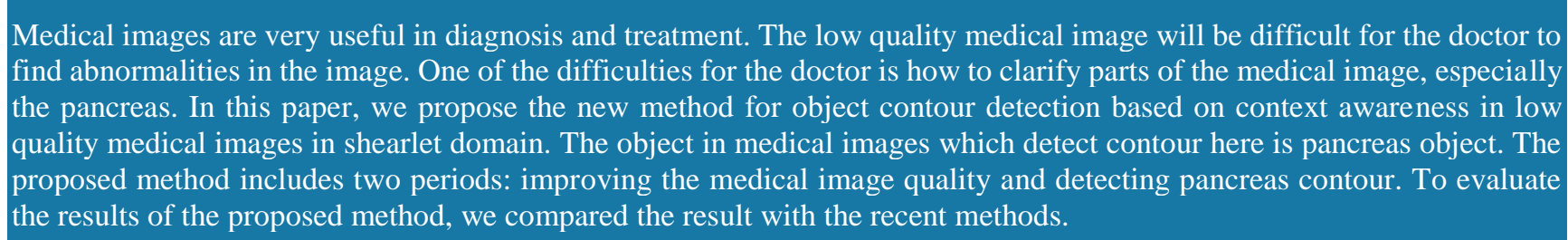

Keywords: B-spline, shearlet transform, low quality image, pancreas detection

Received on 26 June 2018, accepted on 08 July 2018, published on 10 December 2018

Copyright (C) 2018 Nguyen Thanh Binh et al., licensed to EAI. This is an open access article distributed under the terms of the Creative Commons Attribution licence (http://creativecommons.org/licenses/by/3.0/), which permits unlimited use,

distribution and reproduction in any medium so long as the original work is properly cited.

doi: 10.4108/eai.18-6-2018.156319

\section{Introduction}

Medical images are useful for diagnosing and treating diseases. Some diseases are detected through medical images. One of the difficulties for the doctor is how to clarify parts of the medical image, especially the pancreas. The internal organs in the body are often overlapped and obscured. Therefore, the problem of finding the boundary of the object in the medical image has many challenges.

For edge detection, there are many methods which are proposed such as: Canny [1, 2, 3], Sobel [4], B-spline [5, 6,
$7,8,9]$ and many methods using the generation types of wavelet transform [5, 10, 14, 22] for edge detection. Patrick [6] was proposed to be B-spline snakes by multi-scale to apply for contour detection and continued development with Brigger [9]. On the other hand, many previous methods for edge detection are done in transforms as Wang [5], Lei [10], etc. Shimizu [11] proposed pancreas segmentation method from contrast-enhanced multiphase computed tomography (CT). Marius [12] used liver and spleen as starting points for detection of splenic. Shimizu [13] used atlas-guided segmentation and level-sets for pancreas segmentation.

*Corresponding author. Email: ntbinh@hcmut.edu.vn 
Bagci [15] proposed a hybrid segmentation method using the region-based combined with shape-based methods. The method not only works well with CT images but also MR images. Chen [16] segmented multiple organs by combining the active shape modeling and graph cut algorithm. Laishram [17] proposed an edge detection method using Particle Swar Optimization and Fuzzy $\mathrm{C}$ means to segment Canny algorithm to detect edges in input image. Samit [18] proposed an edge detection using ant colony optimization and $\mathrm{F}$ ratio. The method used $\mathrm{F}$ ratio technique to determine the optimum threshold value. Wenshuo [19] proposed an improved Sobel edge detection. The method combined Sobel operator and sof-threshold wavelet de-noising. However, each method has strengths and weaknesses [14].

With low quality medical images, we want to improve the quality of medical images before we detect object contour. In the part time, there are many methods for improving the quality of medical images [25]. The quality of medical images affects to detect edges of the object. When the quality of medical images is not good, the edge detection is hard. Canny [1] proposes a method for edge detection included as removed noise by Gaussian filter and all steps do not include deblurring images to prepare for edge detection.

In this paper, we propose the new method for object contour detection based on context awareness in low quality medical images in shearlet domain. The object in medical images which detect contour here is pancreas object. The proposed method includes two periods: improving the medical image quality and detecting pancreas contour. The rest of the paper is organized as follows: in section 2 , we described shearlet transform and its usefulness for pancreas detection. The details of the proposed method are explained in section 3. The experiment results and conclusion are presented in section 4 and section 5 .

\section{Shearlet transform background for object detection}

Shearlets are one of the types of new generation wavelets transforms. It is similar to curvelets. Both of them perform a multiscale and multidirectional analysis. Shearlet transforms have two different types of shearlet systems: band-limited shearlet system and compactly supported shearlet system [20]. Computational complexity of the band-limited shearlet is high.

The digitization of discrete shearlet transform is performed in the frequency domain. The discrete shearlet transform is of the form [21]:

$$
\begin{gathered}
f \mapsto\left\langle f, \psi_{n}\right\rangle=\left\langle\hat{f}, \hat{\psi}_{n}\right\rangle \\
\left\langle\hat{f}, \hat{\psi}_{n}\right\rangle=\left\langle\hat{f}, 2^{-j \frac{3}{2}} \hat{\psi}\left(s_{k}^{T} A_{4^{-j} .}\right) \exp ^{2 \Pi i^{<A} 4^{-b>}}\right\rangle \text { with } b=j S_{k} m, . . \\
f \mapsto\left\langle\hat{f}, \hat{\psi}_{n}\right\rangle=\left\langle\hat{f}, 2^{-j \frac{3}{2}} \hat{\psi}\left(s_{k}^{T} A_{4}, j .\right) e^{\left.2 \Pi i^{<A} 4^{-j S_{k} m, . .}\right\rangle}\right\rangle
\end{gathered}
$$

where, $\mathrm{n}=(\mathrm{j}, \mathrm{k}, \mathrm{m}, \mathrm{i})$ are indexes - scale $\mathrm{j}$, orientation $\mathrm{k}$, position $\mathrm{m}$ and cone $\mathrm{i}$.
If $\mathrm{f}(\mathrm{x})$ is piecewise $\mathrm{C}^{2}$, the approximation error of reconstruction with $\mathrm{N}$-largest coefficients $\mathrm{f}_{\mathrm{N}}(\mathrm{x})$ in the shearlet expansion is given by [22]:

$$
\left\|f-f_{N}\right\|_{2}^{2} \leq B \cdot N^{-2}(\log N)^{3}, \quad N \rightarrow \infty
$$

We have chosen shearlet transform because of its high directionality and representation of salient features (edges, curves and contours) of the image in a better way compared with the other wavelet transform.

Shearlet transform is useful for object detection due to its following properties [23]:

(i) Frame property: It is helpful for stable reconstruction of image.

(ii) Localization: Each of the shearlet frame elements need to be localized in both space and frequency domain.

(iii) Sparse approximation: It is useful for providing sparse approximation comparable to the band-limited shearlets.

Similar to the curverlet transform, the most essential information of the image is compressed into relatively few large coefficients, which coincides with the area of major spatial activity in shearlet domain.

\section{Object contour detection in low quality medical images}

In this section, we propose a method to detect object contour in low quality medical images. The low quality medical images are the medical images which consist of noise or blur or the both. The proposed method is presented as figure 1 and it includes two periods as: (i) improving the medical image quality and (ii) detecting object contour.

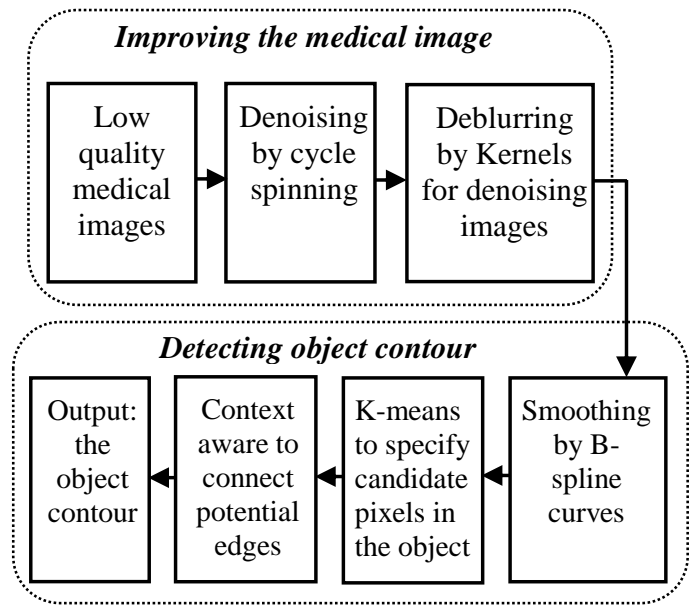

\section{Figure 1. The proposed method for object contour}

We increase the sharpness of edge of objects in images by denoising and debluring. The result of this period is the medical images which consist of strong objects. The output of this period is the input of object contour period.

\subsection{Improvement of the medical image quality in shearlet domain}


The medical image may be consisting of noise or blur or both. The aim of this period is to improve the quality of image but keep the information in the image, avoid loss data in the image. The process of this period is presented in [24].

The shearlet transform is useful for denoising or debluring due to its properties. Firstly, we use cycle spinning for denoising. Secondly, we use Kernels for deblurring in shearlet domain. In here, we summary the steps of this period as [24]:

(i) Define type of shearlet (filter bank) and number of scales in shearlet domain.

(ii) Shearlet decomposition, applying cycle spinning in each direction of shearlet decomposition.

(iii) Denoising processes by threshold:

$$
\text { Threshold }=\frac{1}{2^{j-1}}\left(\frac{\sigma}{\mu}\right) M
$$

where, $\mathrm{j}$ is the number of level at which thresholding is applied. $\mathrm{M}$ is absolute median of shearlet coefficients, $\sigma$ and $\mu$ are standard deviation and absolute mean of shearlet coefficients at $\mathrm{j}$-th level.

(iv) Compare the detail coefficients with threshold values given and make the coefficient values closer to 0 .

(v) Continue with the new novel Kernels set, deblurring medical image does not require to determine the PSF (point spread function). We use the new Kernels set to remove blur out of denoising image by apply matrix $3 \times 3$ : $\{0-10,-15-1,0-10\}$.

The output images of the above steps are removed noise and blur.

\subsection{Object contour detection in low quality medical images.}

The aim of this period is to detect object contour (pancreas object). The period includes three steps as: smoothing by Bspline curves, using K-means to specify candidate pixels in the object and context awareness to connect potential edges.

\section{(i) Smoothing by B-spline curves}

We use a B-spline curve to smooth the image as [25]. A B-spline curve gives more information than Bézier curve. A Bézier curve is a parametric curve. From the set of $(n+1)$ control points $\left(\mathrm{P}_{0}, \mathrm{P}_{1}, \ldots, \mathrm{P}_{\mathrm{n}}\right)$, satisfy: $\mathrm{m}=\mathrm{n}+\mathrm{p}+1$, a degree $\mathrm{p}$ and knot vector $\mathrm{U}=\left\{\mathrm{u}_{0}, \mathrm{u}_{1}, \ldots, \mathrm{u}_{\mathrm{m}}\right\}$. The B-spline curve of degree $\mathrm{p}\left(N_{i, u}(u)\right)$ defined by [25]:

$$
C(u)=\sum_{i=0}^{n} N_{i, p}(u) P_{i}
$$

B-spline with the smoothing function $\beta_{2^{-1}}^{n+1}$ and $\beta_{2^{-1}}^{n+2}$ uses $2^{-1}$ level is:

$$
\psi^{n}(x)=\frac{d^{2}}{d x^{2}} \beta_{2^{-1}}^{n+2}(x)=8\left(\beta^{n+2}\right)^{(2)}(2 x)
$$

where, $\mathrm{n}$ is the order of wavelet transform.

The desirable geometric characteristics of B-Spline curves and surfaces of degree $\mathrm{p}$ are defined as:

$$
N_{i, 0}(u)=\left\{\begin{array}{c}
0 \text { if } u_{i} \leq u \leq u_{i+1} \\
1, \text { otherwise }
\end{array}\right.
$$

$$
N_{i, p}=\frac{u-u_{i}}{u_{i+p}-u_{i}} N_{i, p-1}(u)+\frac{u_{i+p+1}-u_{i}}{u_{i+p+1}-u_{i+1}} N_{i+1, p-1}(u)
$$

To determine gradients of the medical images after the smoothing step, we use the Kernel. The value of gradients (known as the edge strengths) is calculated by Euclidean distance measure.

\section{(ii) Using K-means to specify candidate pixels in the object}

In here, we used the k-means clustering to decide that the pixel in the search region belongs to the object which we find or not [26]. The boundary of the object in the next slice is near its border in the previous slice. We divided all pixels in the search region into two groups: group A and group B. The group A includes the pixel near based on a distance transform feature and otherwise assigned as B. The pixels in the group A are assigned to the pancreas.

\section{(iii) Context awareness to connect potential edges}

In image processing, if a piece of information can be used to characterize the situation of a participant in an interaction, then that information is context [27]. We can define that the context is any information as: pixel, noise, strong edge, and weak edge in a medical image that is considered relevant to the interaction between pixels and pixels including noise, weak and strong edge themselves [27].

After the above section, the problem is how to connect the strong points in object boundary together. Our aim is to connect two points which the distance between them is shortest based on context awareness.

For a set of node points $\mathrm{n}(\mathrm{k})$, where $\mathrm{k}=0,1, \ldots,(\mathrm{N}-1)$, we require that $n(N)=n(0)$ and $n(-1)=n(N-1)$.

The corresponding boundary conditions are periodic. The extended signal ns(k) of infinite length can be described [27] as

$$
n_{s}(k)=n(k \bmod N)
$$

The different choices can be implemented for the open snake such as mirror or anti-mirror boundary conditions. In this application, the anti-mirror conditions with a pivot at the boundary value are the most suitable choice because they allow us to lock the end points of the curve [28].

\section{Experimental and evaluation}

In this section, we present the experiments and the results of the proposed method for pancreas contour detection. Experiments are run in Matlab 2013a and on computer hardware of Intel core i7, 3.2 GHz CPU, 16 GB DDR3 memory. Our dataset has 200 medical images (include 100 low quality and 100 strong low quality medical images) with the sizes $256 \times 256$ and $512 \times 512$ in dataset.

We computed the Jaccard Index (JI) between an extracted region and a true one $[31,32]$, which were manually defined by a medical expert to evaluate the results. The JI value is calculated the follow as: 


$$
J I(X, Y)=\frac{|\mathrm{X} \cap Y|}{|\mathrm{X} \cup \mathrm{Y}|} \times 100
$$

where, $\mathrm{X}$ is extracted region, $\mathrm{Y}$ is true region. If $\mathrm{X}$ and $\mathrm{Y}$ are both empty, we define $\mathrm{JI}(\mathrm{X}, \mathrm{Y})=100$. The index ranges from 0 to $100 \%$, with higher values representing better performance [31].

The strong and weak objects in medical images are defined in [27] as: "the strong object is an object of which boundaries are clear and the weak object is defined as an object of which boundaries are blurred". In here, we base on the concept from [25]: "the blur details include noise details". Consequently, the weak object is an object of which boundaries are blurred and noised.

We test the proposed method and compare the result with other methods such as: Shimizu method [29], Marius method [30] and Nguyen method [14] in the above dataset. In here, we only present two cases as figure 2 and figure 3 .

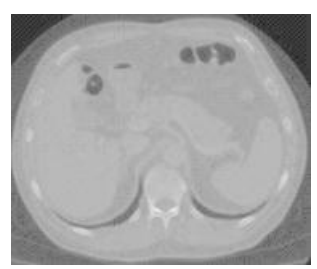

(a)

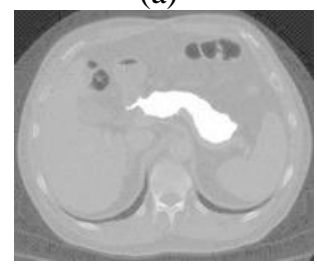

(c)

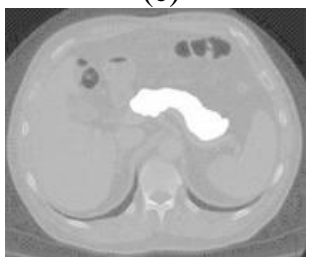

(e)

Figure 2. Results of pancreas detection by proposed method and the other methods in case the low quality medical images.

(a) The original medical image.

(b) Pancreas detection by Shimizu method [29], $(\mathrm{JI}=72.71)$

(c) Pancreas detection by Marius method [30], ( $\mathrm{JI}=74.15)$

(d) Pancreas detection by Nguyen method [14], $(\mathrm{JI}=74.92)$

(e) Pancreas detection by the proposed method, $(\mathrm{JI}=75.16)$

The results of the proposed method with the other methods are presented as figure 2. Figure 2(a) is the original medical image. The result of Shimizu method [29] for pancreas detection is figure 2(b), Marius method [30] is figure 2(c), Nguyen method [14] is figure 2(d) and the proposed method is figure $2(e)$.

The JI value in figure 2(e) is higher than the JI value in figure 2(b), figure 2(c) and figure 2(d). Therefore, the result of the proposed method is better than the other methods.

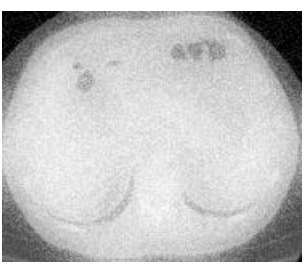

(a)

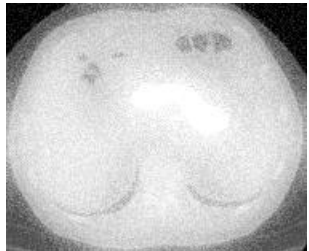

(c)

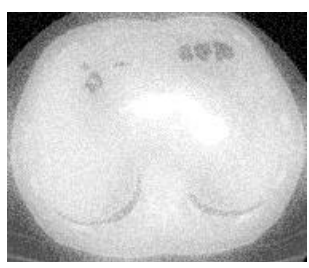

(e)

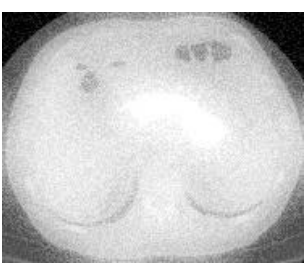

(b)

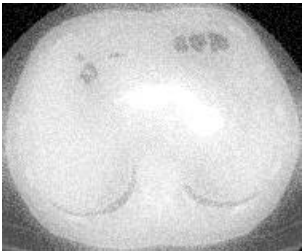

(d)
Figure 3. Results of pancreas detection by proposed method and the other methods in case the strong low quality medical images.

(a) The original medical image.

(b) Pancreas detection by Shimizu method [29], ( $\mathrm{JI}=52.21)$

(c) Pancreas detection by Marius method [30], $(\mathrm{JI}=53.82)$

(d) Pancreas detection by Nguyen method [14], (JI = 54.73)

(e) Pancreas detection by the proposed method, $(\mathrm{JI}=55.16)$

The other experiment is presented as figure 3. The original medical image is presented as figure 3(a). The result of Shimizu method [29] for pancreas detection is figure 3(b), Marius method [30] is figure 3(c), Nguyen method [14] is figure 3(d) and the proposed method is figure 3(e).

In figure 3 , the JI value in figure $3(\mathrm{e})$ is also higher than the JI value in the other figures. Therefore, the result of the proposed method is better than the other methods. The average JI value of all images in dataset is presented in table 1.

Table 1. The average $\mathrm{Jl}$ value of the methods.

\begin{tabular}{|c|c|c|c|c|}
\hline $\begin{array}{c}\text { The } \\
\text { number of } \\
\text { pancreas } \\
\text { images }\end{array}$ & $\begin{array}{c}\text { Average JI } \\
\text { value of } \\
\text { Shimizu } \\
\text { method } \\
{[29]}\end{array}$ & $\begin{array}{c}\text { Average } \\
\text { JI value } \\
\text { of Marius } \\
\text { method } \\
{[30]}\end{array}$ & $\begin{array}{c}\text { Average JI } \\
\text { value of } \\
\text { Nguyen } \\
\text { method } \\
{[14]}\end{array}$ & $\begin{array}{c}\text { Average JI } \\
\text { value of } \\
\text { proposed } \\
\text { method }\end{array}$ \\
\hline $\begin{array}{c}100 \text { images } \\
\text { with low } \\
\text { quality }\end{array}$ & 71.127 & 72.043 & 72.411 & 73.312 \\
\hline $\begin{array}{c}100 \text { images } \\
\text { with strong } \\
\text { low quality }\end{array}$ & 51.841 & 51.946 & 52.024 & 52.927 \\
\hline
\end{tabular}


The results of the proposed method are better than the other methods in the table 1 . As we present in section 2, the shearlet transform perform a multiscale and multidirectional analysis. Shearlet transform is high directionality and representation of salient features (edges, curves and contours) of the image in a better way compared with wavelet transform. Therefore, the medical image quality in shearlet domain is improved. Moreover, the object contour detection period, we use context awareness to connect potential edges. So, these are the reasons of the results of the proposed method which are better than those of the other methods.

\section{Conclusions}

Object contour in the high quality medical images is hard work. With the low quality of medical images, it is more challenging. In the previous object detection algorithms, weak objects detection are a challenge. In this paper, we propose the new method for object contour detection based on context awareness in low quality medical images in shearlet domain. The object in medical images which detect contour here is pancreas object. The proposed method includes two periods: improving the medical image quality and detecting pancreas contour. We test with the dataset which is collected from many hospitals include 200 medical images. We test the proposed method with the above dataset and compare the results with other methods such as: Shimizu method [29], Marius method [30] and Nguyen method [14]. The results of the proposed method are better than the other methods. In the case that the quality of medical images is extremely low quality, then the work of the pancreas contour detection has challenges. Because the information of the image gets lost, image reconstruction is required. In the future works, we extend this proposed method for the extremely low quality medical image case.

\section{Acknowledgements}

This research is funded by Vietnam National University Ho Chi Minh City (VNU-HCM) under grant number C2017-2020.

\section{References}

1. John Canny. A computational approach to edge detection. Pattern Analysis and Machine Intelligence, IEEE Transactions on, Vol. 8, Issue 6, pp. 679-698, 1986

2. R. Deriche. Using Canny's criteria to derive a recursively implemented optimal edge detector. International Journal Computer Vision, Vol. 1, pp. $167-187,1987$

3. Moeslund. Canny edge detection, http://www.cse.iitd.ernet.in/ pkalra/csl783/canny.pdf, 2009, (last accessed: 15 June 2018)
4. O. R. Vincent, O. Folorunso. A descriptive algorithm for sobel image edge detection. Proceedings of Informing Science \& IT Education Conference, 2009

5. Wang Yuping, Cai Yuanlong. Multiscale B-spline wavelet for edge detection. Science in China (Series A), Vol. 38, No. 4, 1995

6. Patrick Brigger, Michael Unser. Multi-scale Bspline Snakes for General Contour Detection. Wavelet Applications in Signal and Image Processing VI, SPIE, Vol 3458, 1998

7. A.D. Bhatt and R.V. Warkhedkar. Reverse engineering of human body: a B-Spline based heterogeneous modeling approach. ComputerAided Design and Applications, Vol. 5, No. 1-4, pp. 194-208, 2008

8. A.D. Bhatt and R.V. Warkhedkar. Material-solid modeling of human body: a heterogeneous B-Spline based approach. Computer-Aided Design, Vol. 41, pp. 586-597, 2009

9. P. Brigger, J. Hoeg and M. Unser. B-Spline snakes: a flexible tool for parametric contour detection. IEEE Transactions on Image Processing, Vol. 9, No. 9, pp. 1484-1496, 2000

10. Lei Zhang, Paul Bao. Edge detection by scale multiplication in wavelet domain. Pattern Recognition Letters 23, Elsevier, pp.1771-1784, 2002

11. Shimizu A, Kimoto T, Kobatake $\mathrm{H}$ et al., Automated pancreas segmentation from three-dimensional contrast-enhanced computed tomography, International Journal of Computer Assisted Radiology and Surgery, Volume 5, Issue 1, pp 8598, 2010

12. Marius Erdt, Matthias Kirschner, Klaus Drechsler, Stefan Wesarg, Matthias Hammon, Alexander Cavallaro, Automatic Pancreas Segmentation In Contrast Enhanced CT Data Using Learned Spatial Anatomy And Texture Descriptors, Biomedical Imaging: From Nano to Macro, 2011 IEEE International Symposium on, pp. 2076 - 2082, 2011

13. Shimizu, R. Ohno, T. Ikegami, H. Kobatake, S. Nawano, and D. Smutek, Segmentation of multiple organs in non-contrast $3 \mathrm{~d}$ abdominal images, Int $\mathbf{J}$ Computer Assisted Radiology and Surgery, Vol. 2, pp. 135-142, 2007

14. Nguyen Huu The, Vo Thi Hong Tuyet, Nguyen Mong Hien, Nguyen Thanh Binh, Pancreas segmentation in medical images based on gaussian mixture model in bandlet domain, The 7th International Conference in Vietnam on the Development of Biomedical Engineering, June 2729, Vietnam, Springer, 2018

15. Bagci U, Chen X, Udupa JK. Hierarchical scalebased multi-object recognition of 3D anatomical structures. IEEE Transactions on Medical Imaging, Vol 31, Issue: 3, pp 777-789, 2012 
16. Chen X, Bagci U. 3D automatic anatomy segmentation based on iterative graph cut ASM Medical Physics, Vol 38, pp 4610 - 4622, 2011

17. Romesh Laishram, Wahengbam Kanan Kumar, Anshuman Gupta, Khairnar Vinayak Prakash, A novel MRI brain edge detection using PSOFCM segmentation and canny algorithm, International Conference on Electronic Systems, Signal Processing and Computing Technologies, pp 398401, 2014

18. Samit Ari Dipak Kumar Ghosh, Prashant Kumar Mohanty, Edge detection using ACO and F ratio, Journal of Signal, Image and Video Processing, Vol. 8, Issue 4, pp 625-634, 2014

19. Wenshuo Gao, Lei Yang, Xiaoguang Zhangand Huizhong Liu, A improved sobel edge detection, 3rd IEEE International Conference on Computer Science and Information Technology, pp. 67-71, 2010

20. Gitta Kutyniok, Demetrio Labate, Shearlets: multiscale analysis for multivariate data, Birkhauser, ISBN 978-0-8176-8316-0, 2012.

21. Wang-Q Lim, The discrete shearlet transform: a new directional transform and compactly supported shearlet frames, IEEE Transactions on image processing, Vol 19, No 5, pp 1166 - 1180, 2010

22. K. Guo, D. Labate, Optimally sparse multidimensional representation using shearlets, SIAM Journal on Mathematical Analysis, vol. 39, pp. 298-318, 2007

23. Vishal M. Patel, Glenn R. Easley, Dennis M. Healy, Shearlet-based deconvolution, IEEE Transactions on Image Processing, Vol. 18, No. 12, pp 26732685, 2009

24. Nguyen Thanh Binh, Vo Thi Hong Tuyet. Improving the quality of medical image in shearlet domain. The 2015 International Conference on Advanced Technologies for Communications, DOI: 10.1109/ATC.2015.7388361, pp 406-410, 2015

25. Vo Thi Hong Tuyet, Nguyen Thanh Binh. Edge detection in low-quality medical images. The 2nd EAI International Conference on Nature of Computation and communication, Vietnam, pp 351362,2016

26. Amir H.F, Yen-Wei C., Reza A. Z, Akira F, Yoshinobu Sato, Masatoshi Hori, Noriyuki Tomiyama. Segmentation of liver in low-contrast images using k-means clustering and geodesic active contour algorithms, IEICE Transactions on Information and Systems, Volume E96.D, Issue 4, pp 798-807, 2013

27. Nguyen Thanh Binh. Image contour based on context aware in complex wavelet domain. Journal of Human-centric Computing and Information Sciences, Vol 5, Issue 1, pp 1-17, 2015

28. Huaizu Jiang, Jingdong Wang, Zejian Yuan, Tie Liu, Nanning Zheng, Shipeng Li. Automatic salient object segmentation based on context and shape prior, British Machine Vision Conference, pp 1-12, 2011

29. Shimizu A, Kimoto T, Kobatake $\mathrm{H}$ et al., Automated pancreas segmentation from three-dimensional contrast-enhanced computed tomography, International Journal of Computer Assisted Radiology and Surgery, Volume 5, Issue 1, pp 8598, 2010

30. Marius Erdt, Matthias Kirschner, Klaus Drechsler, Stefan Wesarg, Matthias Hammon, Alexander Cavallaro, Automatic Pancreas Segmentation In Contrast Enhanced CT Data Using Learned Spatial Anatomy And Texture Descriptors, IEEE International Symposium on Biomedical Imaging: From Nano to Macro, 2011, pp. 2076 - 2082, 2011

31. Tran Duc Tam, Nguyen Thanh Binh. Efficient pancreas segmentation in computed tomography based region growing. Proceedings of the International Conference on Nature of Computation and Communication, pp 332-340, 2014.

32. R Real, JM Vargas, The probabilistic basis of Jaccard's index of similarity, Systematic Biology, Vol. 45, No. 3, 1996 